\title{
Research Square

\section{Value of Radiomics Features From Adrenal Gland and Periadrenal Fat in CT Images for Predicting COVID-19 Prognosis}

\author{
Mudan zhang \\ Guizhou Medical University \\ Xuntao Yin \\ Guangzhou Woman and Children's medical centra \\ Wuchao Li \\ Guizhou Provincial People's Hospital
}

\section{Yan Zha}

Guizhou Provincial People's Hospital

\section{Xianchun Zeng}

Guizhou Provincial People's Hospital

\section{Xiaoyong Zhang}

Guizhou Provincial People's Hospital

\section{Jingjing Cui}

Shanghai United Imaging intelligence CO.LTD

\section{Zhong Xue}

Shanghai United Imaging Intelligence CO.LTD

\section{Rongpin Wang}

Guizhou Provincial People's Hospital

Chen Liu ( $\sim$ liuchen@aifmri.com )

Southwest Hospital, Third Military Medical University (Army Medical University) https://orcid.org/0000-0001-5149-2496

\section{Research}

Keywords: adrenal gland, periadrenal fat, auto-segmentation, COVID-19, radiomics

Posted Date: November 10th, 2021

DOI: https://doi.org/10.21203/rs.3.rs-989736/v1

License: (c) (i) This work is licensed under a Creative Commons Attribution 4.0 International License. Read Full License 


\section{Abstract}

Background: Endocrine system plays an important role in infectious disease prognosis. Our goal is to assess the value of radiomics features extracted from adrenal gland and periadrenal fat CT images in predicting disease prognosis in patients with COVID-19.

Methods: A total of 1,325 patients (765 moderate and 560 severe patients) from three centers were enrolled in the retrospective study. We proposed a 3D cascade V-Net to automatically segment adrenal glands in onset CT images. Periadrenal fat areas were obtained using inflation operations. Then, the radiomics features were automatically extracted. Five models were established to predict the disease prognosis in patients with COVID-19: a clinical model (CM), three radiomics models (adrenal gland model [AM], periadrenal fat model [PM], fusion of adrenal gland and periadrenal fat model [FM]), and a radiomics nomogram model (RN).Data from one center (1,183 patients) were utilized as training and validation sets. The remaining two (36 and 106 patients) were used as 2 independent test sets to evaluate the models' performance.

Results: The auto-segmentation framework achieved an average dice of 0.79 in the test set. CM, AM, PM, FM, and RN obtained AUCs of $0.716,0.755,0.796,0.828$, and 0.825 , respectively in the training set, and the mean AUCs of $0.754,0.709,0.672,0.706$ and 0.778 for 2 independent test sets. Decision curve analysis showed that if the threshold probability was more than $0.3,0.5$, and 0.1 in the validation set, the independent-test set 1 and the independent-test set 2 could gain more net benefits using RN than FM and $\mathrm{CM}$, respectively.

Conclusion: Radiomics features extracted from CT images of adrenal glands and periadrenal fat are related to disease prognosis in patients with COVID-19 and have great potential for predicting its severity.

\section{Key Summary Points}

1. Endocrine system plays an important role in infectious disease prognosis, however there is lack of quantitative studies showing the effect of adrenal glands or periadrenal fat on the prognosis of patients with COVID-19.

2. We hypothesis radiomics features extracted from CT images of adrenal glands and periadrenal fat are related to disease prognosis in patients with COVID-19 and have great potential for predicting its severity.

3. We proposed a 3D cascade V-Net to automatically segment adrenal glands in onset CT images, and their periadrenal fat areas were obtained using inflation operations. The auto-segmentation framework achieved an average Dice of 0.79 in the test set.

4. Radiomics nomogram obtained AUCs of 0.825 in the training set, and the mean AUCs of 0.778 for 2 independent test sets for classifying moderate and severe cases.

\section{Introduction}

Coronavirus disease 2019 (COVID-19) has caused serious public health problems, much worse than those caused by SARS in 2002-2003. Until 18 June, 2020, WHO reported 176,945,596 confirmed patients and 3,836,828 deaths globally (https://covid19.who.int/). Although a suitable diagnosis and treatment protocol for the national situation has been enacted in each country, separating moderate patients from severe patients who need special care still lack effective strategies. Thus, predicting early disease prognosis and accurately identifying patients at risk of developing a severe or critical disease is urgently required. This is not only conducive to the early disease control but also able to effectively allocate medical resources globally.

Because it is unclear that whether the polymerase chain reaction (PCR) technique [1], clinical symptoms, and laboratory tests are correlated with prognosis in patients with COVID-19 [2], some researchers studied prognosis of COVID-19 by combining artificial intelligence (Al) technology and CT images. Zhang et al. [3] constructed a comprehensive system for accurate diagnosis, quantitative analysis, and prognoses of COVID-19 pneumonia, and they proved that the combination of chest CT and $\mathrm{Al}$ technology can potentially predict disease prognosis in patients with COVID-19. While most studies focusing on the relationship between image features and disease prognosis, they ignored the endocrine system that plays an important role in disease prognosis [4]. In fact, this was also not paid attention during SARS outbreak [5], and it is until recently that researchers found that damage and changes in endocrine functions were important predictors of morbidity and mortality in SARS patients [6]. However,

Page 2/16 
there is lack of quantitative studies showing the effect of adrenal glands or periadrenal fat on the prognosis of patients with COVID-19.

The adrenal gland is a key component of the body's stress and immune system [7]. The adrenal gland integrates the cortex, which secretes glucocorticoids plus catecholamines, and the medulla secreting catecholamines within one organ capsule. The pituitary gland mostly regulates adrenal cortex function through adrenocorticotropic hormone (ACTH) and aldosterone, playing an important role in the renin-angiotensin system. The adrenal medulla also secretes catecholamines in response to the sympathetic nervous system (SNS). The entire system and the organs mentioned above are closely related to the immune-inflammatory state of COVID-19 patients [8]. It is known that the adrenal gland changes under two conditions. Firstly, adrenal gland could be attacked from SARS-CoV-2 through angiotensin-converting enzyme-2 (ACE2) pathway [9]. Secondly, the organ adapts to physiological needs and pathological conditions due to the astonishing regenerative capacity and adjusts to unique micro-environments relying on an extensive vascular network [10]. Therefore, we hypothesize that adrenal gland may change and adapt to SARS-CoV-2 invasion. The periadrenal fat could be bounded to be affected as the closest tissue around the adrenal gland.

Actually, the subtle changes of the adrenal gland can be detected by extracting radiomics features from chest CT images using Al technology. Radiomics is an emerging technique that can convert images difficult to be distinguished by human into highthroughput quantitative features that may reflect potential pathological and physiological states [11-12]. The segmentation of the region of interest $(\mathrm{ROI})$ is generally the first and key step to constructing a radiomics model, but it is time-consuming and errorprone due to repeated labor in manual operations. Combining the auto-segmentation framework based on Al technology and manual revision can improve both efficiency and quality for ROI segmentation [13]. Cascaded V-Nets have been used to segment brain tumors with satisfactory results which could be further improved if extra information, such as coarse localization, is incorporated [14].

To study the relationship between radiomics features extracted from the adrenal gland and periadrenal fat from CT images and the prognosis in patients with COVID-19, we constructed an auto-segmentation framework and developed a radiomics nomogram (RN) to predict prognosis from CT images by combining clinical indicators. Five models were established and compared to predict the disease prognosis in patients with COVID-19 in order to analyze which feature plays the important role: a clinical model (CM), three radiomics models (adrenal gland model [AM], periadrenal fat model [PM], fusion of adrenal gland and periadrenal fat model [FM]), and a radiomics nomogram model $(\mathrm{RN})$.

\section{Methods}

\section{Ethics Committee Approval and Patient Consent}

This multicenter study was approved by the ethics committees of all the participating hospitals (2020, NO.01). Because of its retrospective nature, informed consent forms were not required but all the image and clinical data were anonymized, and the results of the study did not alter any treatment plans for the subjects. The study was performed according to the principles of the declaration of Helsinki.

\section{Data resources and grouping}

CT images and clinical data were retrospectively collected from three hospitals: Huoshenshan Hospital $(H S H)(n=1,183)$, Maternal and Child Health Hospital Optical Valley Branch Hospital of Hubei province $(\mathrm{MCH})(\mathrm{n}=36)$ and Taikang Tongji (Wuhan) Hospital $(T T H)(n=106)$, China. Diagnosis and clinical classification of patients with COVID-19 were confirmed according to the Diagnosis and Treatment Protocol for Novel Coronavirus Pneumonia (Trial Version 8). Patients of age $\leq 14$ years $(n=2)$ were excluded. All images were non-enhanced chest CT images and reconstructed at a slice thickness of $1.00 \mathrm{~mm}$, the details of CT characteristics are listed in Supplementary Table 1. Patients with adrenal lesions were excluded after being evaluated by two radiologists with more than 10 years experiences. We chose chest CT images scanned within four days to the first diagnosis as the onset image. If the CT scan was done more than once, we chose the one closest to the admission date. Supplementary Figure 1 demonstrates the inclusion and exclusion criteria. 
By dividing the CT images of 1,183 patients from Huoshenshan hospital into the training set $(946,80 \%)$ and the validation set $(237,20 \%)$ randomly, we constructed the prediction models. We used different imaging and clinical features and their combinations to predict the prognosis of COVID-19. Based on the features, we constructed five models: adrenal gland radiomics model (AM), periadrenal fat radiomics model (PM), a fusion of the adrenal gland and periadrenal fat radiomics model (FM), clinical model (CM) and radiomics nomogram (RN). A total of 36 patients (13 moderate and 23 severe patients) from $\mathrm{MCH}$ were included as the independent test set 1 , and 132 patients (113 moderate and 19 severe patients) from TTH were included as the independent test set 2. The workflow of our study is shown in Figure 1.

\section{Auto-segmentation framework and ROls delineation}

The bilateral adrenal glands and the periadrenal fat are used for the study, which need to be segmented from the CT image. First, an experienced radiologist (Y.F.) manually delineated the ROIs of bilateral adrenal glands to acquire the ground truth labels of adrenal, which was delineated according to the image delineating principles of BraTS 2018. Then, a cascaded V-net network was applied to segment the bilateral adrenal glands automatically. The auto-segmentation framework was composed by two V-Nets. We first trained a coarse localization model for coarse segmentation and localization of the adrenal gland area. The second V-Net model was used for defining fine segmentation, and the left and the right adrenal gland regions were obtained. Subsequently, ROIs of periadrenal fat were obtained using morphological inflation algorithm.

The main structure of V-Net includes Down-Blocks and Up-Blocks. Firstly, the high-level context information was extracted by convolutions and Down-Blocks. Skip connections were used to fuse high-level context information and fine-grained local information. Normalization was performed before the images were input to the network. The segmentation frame is shown in Figure 2.

ROIs including bilateral adrenal glands and periadrenal fat from all CT images without annotation were segmented using the autosegmentation framework. Specially, the ROIs with serious nonconformity were manually revised by two radiologists with more than 5 years clinical experiences and validated by another radiologist with 20 years clinical experiences.

\section{Radiomics feature selection and models building}

Radiomics features were extracted from the ROIs on the CT images using the Python package (PyRadiomics V3.0) [15]. B-Spline interpolation resampling was used to normalize the voxel size, and the anisotropic voxels were resampled to form isotropic voxels of $1.0 \mathrm{~mm} \times 1.0 \mathrm{~mm} \times 1.0 \mathrm{~mm}$ before feature extraction. A total of 2,264 features were extracted from each ROI, which can be divided into 3 groups, 450 first-order features, 14 shape features, and 1,800 texture features (gray-level co-occurrence matrix $[G L C M]=525$, gray-level run-length matrix $[G L R L M]=350$, gray-level size zone matrix $[G L S Z M]=400$, gray-level dependence matrix $[G L D M]=400$ and neighboring Gray Tone Difference Matrix [NGTDM] = 125). Image normalization was applied before extracting the radiomics features.

To reduce the dimension and to select important features, we select the most relevant features by the following steps. First, a univariate analysis named K-best was employed. It selected features through P-value calculated by analysis of variance (ANOVA), the features that are significantly different between the severe and moderate groups $(P<0.05)$ were selected. Next, the least absolute shrinkage and selection operator (LASSO) method was used to extract the most informative radiomics features to prevent the curse of dimensionality. It should be noted that Z-score was used to standardize the features before the LASSO algorithm. After feature extraction and selection, three radiomics models (AM, PM and FM) were constructed using logistic regression (LR) algorithm for predicting the disease prognosis of COVID-19. In the FM model, the RadScore of the patient was calculated according to the LASSO algorithm.

\section{RN construction and evaluation}

A univariate analysis was used to assess the relationship between clinical factors and serum biomarkers with disease outcome. The clinical factors and serum biomarkers with $P<0.05$ were introduced into multivariate LR to select the best combination of features for the prediction model. To predict the disease prognosis of COVID-19, we applied the multivariate LR model to build CM using clinical indicators and RN using the RadScore from FM with clinical indicators. 
The multi-collinearity among the RadScore and valuable clinical indicators in RN was evaluated. Specially, we achieved this by calculating the variance inflation factor (VIF) through collinearity diagnosis. In the end, RN was verified in the validation and two independent-test sets. Calibration curves and Hosmer-Lemeshow test were used to assess the relationship between the predicted risks and actual results. Decision curve analysis (DCA) and calibration curve were used to evaluate the performance of the RN.

\section{Statistics}

Before building the models, differences in clinical factors and serum biomarkers between moderate and severe patient sets were assessed using Mann-Whitney $U$ test or Student's $t$-test for continuous variables and the $\chi 2$ test or Fisher's exact test for categorical variables. All the data were processed by SPSS with Windows version 26.0 (IBM Corp., Armonk, New York, USA). P< 0.05 was considered a statistically significant difference.

Dice coefficient was used to assess the effectiveness of auto-segmentation framework. The area under curve (AUC) of receiveroperating characteristics (ROC) with $95 \%$ confidence interval $(95 \% \mathrm{Cl})$, the accuracy, the sensitivity, and the specificity were used to evaluate the performance of AM, PM, FM, CM and RN. Significant differences in AUC values among different models were estimated using the DeLong test.

\section{Results}

\section{Patient characteristics}

A total of 1,351 patients (789 moderate and 562 severe patients) were enrolled in the retrospective study from three hospitals (1,183 patients from $\mathrm{HSH}, 36$ patients from $\mathrm{MCH}$ and 132 from TTH). The data of 1,183 patients from HSH were formed as training set (946 patients) and validation set (237 patients), including 663 patients who suffered from moderate COVID-19 and 520 who suffered from severe COVID-19. Among all the data, 687 Patients were diagnosed as moderate pneumonia at first admission and 24 patients among them changed to severe during the stay, 496 patients were diagnosed as severe at first admission but only one severe patient changed to moderate.

The patient characteristics in training and validation sets are listed in Table 1. No significant differences were observed between the training and validation set in age $(P=0.273)$ and sex $(P=0.694)$. In addition, Interleukin $6(\mathrm{IL}-6)$ levels, lymphocyte count $(\mathrm{L})$, Creactive protein (CRP), procalcitonin (PCT), D dimer (DD), glutamic oxaloacetic transaminase (AST), B-type natriuretic peptide (BNP), lactate dehydrogenase (LDH), creatine kinase (CK), and creatine kinase-MB (CK-MB) differed significantly between moderate and severe pneumonia sets both in training and validation sets $(P<0.05)$. 
Table 1

Clinical characteristics of patients in training and validation set $(n=1183)$.

\begin{tabular}{|c|c|c|c|c|c|c|c|}
\hline \multirow[t]{2}{*}{ Variable } & \multirow[b]{2}{*}{$\begin{array}{l}\text { Moderate } \\
\text { pneumonia }\end{array}$} & \multicolumn{3}{|l|}{$\begin{array}{l}\text { Training set } \\
(n=946)\end{array}$} & \multicolumn{3}{|l|}{$\begin{array}{l}\text { Validation set } \\
(n=237)\end{array}$} \\
\hline & & $\begin{array}{l}\text { Severe } \\
\text { pneumonia }\end{array}$ & $P$ & $\begin{array}{l}\text { Moderate } \\
\text { pneumonia }\end{array}$ & $\begin{array}{l}\text { Severe } \\
\text { pneumonia }\end{array}$ & $P$ & $P$ \\
\hline $\begin{array}{l}\text { Age } \\
\text { (yr,mean } \pm S D\end{array}$ & $57.393 \pm 13.753$ & $63.885 \pm 12.189$ & 0.000 & $57.765 \pm 11.862$ & $62.971 \pm 13.094$ & 0.263 & 0.273 \\
\hline \multirow[t]{2}{*}{ Sex n(\%) } & & & 0.213 & & & 0.069 & 0.694 \\
\hline & & & & & \multicolumn{3}{|c|}{$\wedge$} \\
\hline Men & $266(50.1 \%)$ & $226(54.3 \%)$ & & $64(48.5 \%)$ & $54(51.9 \%)$ & & \\
\hline women & $265(49.9 \%)$ & $190(45.7 \%)$ & & $68(51.5 \%)$ & $50(48.1 \%)$ & & \\
\hline IL-6 & $3.051 \pm 4.692$ & $14.400 \pm 97.389$ & 0.000 & $2.865 \pm 3.586$ & $13.323 \pm 49.298$ & 0.000 & 0.768 \\
\hline WBC(109/L) & $5.825 \pm 1.808$ & $6.431 \pm 2.535$ & 0.000 & $6.191 \pm 2.188$ & $6.355 \pm 2.294$ & 0.431 & 1.359 \\
\hline $\mathrm{L}\left(10^{9} / \mathrm{L}\right)$ & $1.607 \pm 0.604$ & $1.368 \pm 0.667$ & 0.064 & $1.577 \pm 0.544$ & $1.350 \pm 0.634$ & 0.040 & 0.568 \\
\hline $\mathrm{N}(109 / \mathrm{L})$ & $3.613 \pm 1.574$ & $4.437 \pm 2.479$ & 0.000 & $3.980 \pm 1.970$ & $4.370 \pm 2.225$ & 0.196 & 0.144 \\
\hline $\mathrm{HB}(\mathrm{g} / \mathrm{L})$ & $125.254 \pm 16.705$ & $117.510 \pm 23.988$ & 0.000 & $123.720 \pm 16.022$ & $117.872 \pm 21.662$ & 0.100 & 0.670 \\
\hline RBC(1012/L) & $4.059 \pm 0.535$ & $3.874 \pm 0.594$ & 0.335 & $3.992 \pm 0.540$ & $3.844 \pm 0.573$ & 0.587 & 0.408 \\
\hline $\operatorname{PLT}\left(10^{9} / \mathrm{L}\right)$ & $235.919 \pm 76.644$ & $232.786 \pm 80.199$ & 0.422 & $247.333 \pm 81.926$ & $227.760 \pm 98.226$ & 0.601 & 2.836 \\
\hline $\mathrm{CRP}(\mu \mathrm{g} / \mathrm{L})$ & $3.604 \pm 3.608$ & $5.467 \pm 4.505$ & 0.000 & $3.367 \pm 3.494$ & $5.463 \pm 4.059$ & 0.000 & 0.259 \\
\hline PCT $(\mu \mathrm{g} / \mathrm{L})$ & $1.794 \pm 2.615$ & $1.456 \pm 2.409$ & 0.000 & $1.720 \pm 2.588$ & $1.351 \pm 2.333$ & 0.006 & 1.331 \\
\hline PT(s) & $11.847 \pm 2.693$ & $12.565 \pm 4.911$ & 0.474 & $11.934 \pm 2.788$ & $12.557 \pm 3.264$ & 0.259 & 0.147 \\
\hline $\mathrm{TT}(\mathrm{s})$ & $13.777 \pm 3.584$ & $14.802 \pm 5.185$ & 0.054 & $13.987 \pm 3.627$ & $14.444 \pm 3.617$ & 0.514 & 0.125 \\
\hline FIB (g/L) & $3.412 \pm 1.068$ & $3.487 \pm 0.961$ & 0.084 & $3.362 \pm 1.056$ & $3.448 \pm 1.035$ & 0.008 & 0.048 \\
\hline $\mathrm{DD}(\mathrm{mg} / \mathrm{L})$ & $1.285 \pm 1.922$ & $1.973 \pm 3.319$ & 0.000 & $1.360 \pm 2.109$ & $2.290 \pm 2.919$ & 0.017 & 0.743 \\
\hline $\mathrm{BS}(\mathrm{mmol} / \mathrm{L})$ & $5.455 \pm 2.029$ & $6.063 \pm 2.736$ & 0.000 & $5.451 \pm 2.137$ & $5.990 \pm 2.820$ & 0.126 & 0.480 \\
\hline ALT (u/L) & $32.753 \pm 34.282$ & $32.750 \pm 29.298$ & 0.975 & $30.552 \pm 31.704$ & $41.450 \pm 52.035$ & 0.048 & 0.069 \\
\hline AST (u/L) & $23.311 \pm 13.339$ & $27.209 \pm 28.423$ & 0.000 & $21.962 \pm 19.107$ & $29.462 \pm 20.117$ & 0.022 & 0.819 \\
\hline $\mathrm{TP}(\mathrm{g} / \mathrm{L})$ & $65.204 \pm 8.089$ & $63.332 \pm 9.042$ & 0.052 & $64.859 \pm 7.912$ & $64.245 \pm 6.695$ & 0.529 & 0.024 \\
\hline $\operatorname{ALB}(\mathrm{g} / \mathrm{L})$ & $37.910 \pm 4.993$ & $35.726 \pm 5.433$ & 0.099 & $37.629 \pm 4.809$ & $36.234 \pm 4.356$ & 0.398 & 0.998 \\
\hline $\begin{array}{l}\text { TBil } \\
(\mu \mathrm{mol} / \mathrm{L})\end{array}$ & $10.337 \pm 6.448$ & $10.697 \pm 5.944$ & 0.752 & $10.170 \pm 4.408$ & $11.957 \pm 8.098$ & 0.024 & 1.233 \\
\hline
\end{tabular}

Clinical characteristics and serum biomarkers of patients in the training and internal validation set. IL-6 = Interleukin 6; WBC=white blood cells count, L=lymphocyte count, $\mathrm{N}=$ neutrophil count, $\mathrm{HB}=$ hemoglobin; $\mathrm{RBC}=$ red blood cell; PLT = blood platelet count; $\mathrm{CRP}=\mathrm{C}$ reactive protein; $\mathrm{PCT}=$ procalcitonin; $\mathrm{PT}=$ prothrombin Time; $\mathrm{TT}$ = thrombin time; $\mathrm{FIB}=$ fibrinogen; $\mathrm{DD}=$ $\mathrm{D}$ dimer, $\mathrm{BS}$ = blood sugar, $\mathrm{ALT}$ = alanine transaminase; $\mathrm{AST}$ = glutamic oxaloacetic transaminase; $\mathrm{TP}=$ total protein; $\mathrm{ALB}=$ albumin; TBil = total bilirubin; D-Bil = direct bilirubin; BUN = blood urea nitrogen; Cre =creatinine; BNP = B-type natriuretic peptide; $\mathrm{Mb}=$ myoglobin; $\mathrm{Tn}$ = troponin; $\mathrm{LDH}=$ lactate dehydrogenase; $\mathrm{CK}=$ creatine kinase; $\mathrm{CK}-\mathrm{MB}=$ creatine kinase-MB.

Note: P-value $<0.05$ means a significant difference. Differences in clinical factors and serum biomarkers between moderate and severe patient sets are assessed using Mann-Whitney $U$ test or Student's $t$ test for continuous variables depending on the normal test and the $\chi 2$ test or Fisher's exact test for categorical variables. 


\begin{tabular}{|c|c|c|c|c|c|c|c|}
\hline Variable & & $\begin{array}{l}\text { Training set } \\
(n=946)\end{array}$ & & & $\begin{array}{l}\text { Validation set } \\
(n=237)\end{array}$ & & \\
\hline $\begin{array}{l}\text { D-Bil } \\
(\mu \mathrm{mol} / \mathrm{L})\end{array}$ & $3.710 \pm 3.996$ & $4.152 \pm 3.791$ & 0.235 & $3.591 \pm 1.962$ & $4.763 \pm 5.033$ & 0.007 & 0.083 \\
\hline $\begin{array}{l}\text { BUN } \\
(\mathrm{mmol} / \mathrm{L})\end{array}$ & $4.609 \pm 2.024$ & $5.119 \pm 2.443$ & 0.000 & $4.713 \pm 1.542$ & $4.972 \pm 1.972$ & 0.153 & 0.506 \\
\hline Cre $(\mu \mathrm{mol} / \mathrm{L})$ & $67.148 \pm 30.737$ & $68.861 \pm 24.245$ & 0.230 & $68.086 \pm 22.061$ & $67.887 \pm 22.575$ & 0.668 & 0.252 \\
\hline BNP (pg/ml) & $13.501 \pm 38.781$ & $39.294 \pm 103.015$ & 0.000 & $20.801 \pm 108.036$ & $48.126 \pm 132.048$ & 0.011 & 6.205 \\
\hline $\mathrm{Mb}(\mu \mathrm{g} / \mathrm{L})$ & $6.900 \pm 10.081$ & $15.497 \pm 112.880$ & 0.003 & $7.362 \pm 8.274$ & $15.685 \pm 15.685$ & 0.004 & 0.000 \\
\hline $\operatorname{Tn}(\mu \mathrm{g} / \mathrm{L})$ & $3.386 \pm 2.797$ & $2.954 \pm 2.848$ & 0.000 & $3.072 \pm 2.846$ & $2.475 \pm 2.830$ & 0.361 & 2.995 \\
\hline $\mathrm{LDH}(\mathrm{u} / \mathrm{L})$ & $176.357 \pm 55.930$ & $223.928 \pm 103.029$ & 0.000 & $193.202 \pm 58.210$ & $175.748 \pm 72.622$ & 0.003 & 0.479 \\
\hline CK (u/L) & $56.329 \pm 47.304$ & $59.656 \pm 79.253$ & 0.013 & $58.540 \pm 50.356$ & $82.177 \pm 126.670$ & 0.001 & 12.009 \\
\hline CK-MB (u/L) & $8.987 \pm 6.190$ & $10.750 \pm 8.914$ & 0.000 & $9.653 \pm 6.454$ & $11.906 \pm 11.724$ & 0.004 & 3.058 \\
\hline \multicolumn{8}{|c|}{$\begin{array}{l}\text { Clinical characteristics and serum biomarkers of patients in the training and internal validation set. IL-6 = Interleukin 6; } \\
\text { WBC=white blood cells count, L=lymphocyte count, } N=\text { =neutrophil count, } \mathrm{HB}=\text { hemoglobin; } \mathrm{RBC}=\text { red blood cell; } \mathrm{PLT}=\mathrm{blood} \\
\text { platelet count; CRP=C reactive protein; PCT = procalcitonin; PT = prothrombin Time; TT = thrombin time; FIB = fibrinogen; DD = } \\
\text { D dimer, BS = blood sugar, ALT = alanine transaminase; AST = glutamic oxaloacetic transaminase; TP = total protein; ALB = } \\
\text { albumin; TBil = total bilirubin; D-Bil = direct bilirubin; BUN = blood urea nitrogen; Cre =creatinine; BNP = B-type natriuretic } \\
\text { peptide; } \mathrm{Mb}=\text { myoglobin;Tn = troponin; LDH= lactate dehydrogenase; CK= creatine kinase; CK-MB = creatine kinase-MB. }\end{array}$} \\
\hline \multicolumn{8}{|c|}{$\begin{array}{l}\text { Note: P-value }<0.05 \text { means a significant difference. Differences in clinical factors and serum biomarkers between moderate } \\
\text { and severe patient sets are assessed using Mann-Whitney U test or Student's t test for continuous variables depending on the } \\
\text { normal test and the } \chi 2 \text { test or Fisher's exact test for categorical variables. }\end{array}$} \\
\hline
\end{tabular}

The auto-segmentation framework, radiomics feature extraction, feature selection, and predicting model construction were all established on the uAI Research Portal V1.1 (Shanghai United Imaging Intelligence, Co., Ltd.).

\section{Adrenal gland and periadrenal fat auto-segmentation}

For adrenal gland segmentation, we manually delineated bilateral adrenal glands from the CT images of 315 patients, 265 of them were used for training, and the rest were used to evaluate the performance. The segmentation model yielded average Dice of $79.48 \%$ for the left adrenal gland and $78.55 \%$ for the right. By average, an average Dice of $79.02 \%$ was achieved. Representative auto-segmentation results are shown in Supplementary Figure 2 . We also visually verified the model by proving segmentation results to the radiologists and acquired satisfactory feedback. The segmentation algorithm was then used to segment all the remaining data automatically.

\section{Radiomics feature and clinical indicator selection}

In the training set, ANOVA and LASSO analyses were used to select the radiomics features most relevant to the prognosis of COVID-19. To build AM, the number of radiomics features was reduced to 45, including 10 first-order features and 35 texture features $(G L C M=5, G L S Z M=19$, GLRLM = 4, GLDM = 5 and NGTDM = 2). In addition, 71 features for PM include 21 first-order features and 50 texture features ( $G L C M=14, G L S Z M=22, G L R L M=4, G L D M=6$ and NGTDM = 4), while 68 features for FM are composed of 12 first-order features and 56 texture features (GLCM=12, GLSZM=31, GLRLM = 4, GLDM=6 and NGTDM =3).

A total of 30 clinical factors and serum biomarkers were analyzed in our study. They were age, sex, IL-6, white blood cell count (WBC), L, neutrophil count (N), hemoglobin (HB), red blood cell count (RBC), blood platelet count (PLT), CRP, PCT, prothrombin time (PT), thrombin time (TT), fibrinogen (FIB), DD, blood sugar (BS), Alanine transaminase (ALT), AST, total protein (TP), albumin(ALB), total bilirubin (TBil), direct bilirubin (D-Bil), blood urea nitrogen (BUN), creatinine (Cre), BNP, myoglobin (Mb), troponin (Tn), LDH, 
creatine kinase (CK), and CK-MB (Table 1).Among them, 17 clinical factors and serum biomarkers were selected using univariate logistic regression analysis, 7 indicators, $L D H, L, H B, D D, W B C, T T$, and TP, were selected using multivariate logistic regression analysis. The relationship between RadScore from FM used in the construction of RN and 7 clinical factors combing serum biomarkers were analyzed using Pearson correlation in training, validation, and two independent-test sets. The difference in RadScores with clinical factors or serum biomarkers was not significant. Supplementary Figure 3 indicates that the radiomics information extracted from onset CT images belonged to another dimension, and this information was not affected by clinical factors and serum biomarkers.

\section{Three radiomics models and clinical model building}

We developed three radiomics models (AM, PM, and FM) based on radiomics features and $\mathrm{CM}$ based on the seven selected independent predictive clinical indicators. We used three evaluation indicators (AUC, 95\% Cl, sensitivity [SEN] and specificity [SPE]) to assess AM, PM, FM, and CM for predicting prognosis of patients with COVID-19 in all sets. In general, AM achieved an AUC of $0.755,0.655,0.716$ and 0.701 in the training set, validation set and 2 independent-test sets, respectively; PM achieved an AUC of 0.796, 0663, 0.692 and 0.652; FM achieved an AUC of 0.828, 0.704, 0.702 and 0.709; CM obtained an AUC of $0.716,0.720,0.716$ and 0.792 (Figure 3 , supplementary table 2 ).

Box-plots summarized the RadScores and seven clinical indicators in training, validation, and two independent test sets, which directly demonstrated the difference of RadScore and seven clinical indicators between the moderate and severe patient sets (Supplementary Figure 4).

\section{RN construction and validation}

Multivariate analysis revealed that RadScore and seven clinical indicators were significant independent factors in predicting disease prognosis of patients with COVID-19. Using collinearity diagnosis, VIF for the radiomics score and seven clinical indicators were from 1.058 to 1.332, indicating no severe collinearity in these factors. Besides, we used the RadScore from FM and seven clinical indicators to construct the RN to assess disease prognosis in patients with COVID-19 (Figure 4). The RN showed satisfactory performance for predicting and assessing the prognosis in patients with COVID-19. The results yield AUC of 0.825 ( $95 \% \mathrm{Cl}, 0.799$ to 0.849$)$ in the training set, $0.736(95 \% \mathrm{Cl}, 0.675$ to 0.791$), 0.749(95 \% \mathrm{Cl}, 0.577$ to 0.878$)$ and $0.806(95 \% \mathrm{Cl}, 0.718$ to 0.877 ) in the validation set, the independent-test set 1 and the independent-test set 2, respectively. (Figure 3 , supplementary table 2).

DeLong's test was used to compare the AUCs of the three radiomics models, $\mathrm{CM}$ and RN, in the training set. The result showed that the AM, PM, FM and RN were significantly better than $\mathrm{CM}(P<0.05)$ and the difference between FM and RN was not statistically significant $(P=0.699)$ in the training set. The calibration curve showed an agreement between the predicted and actual values. The Hosmer-Lemeshow test was not significant in the validation set (mean absolute error [MAE] $=0.029$ ), independent-test set 1 (MAE $=0.072)$ and independent-test set $2(\mathrm{MAE}=0.048)$, which suggests that there was no significant departure from actual values (Supplementary Figure 5). DCA was used to evaluate the performance of RN (Supplementary Figure 6). The RN could get more net benefits than FM and CM with a threshold probability higher than 0.3 in the validation set. Similarity, RN can still get more net benefits than FM and $\mathrm{CM}$ with a threshold probability was more than 0.5 in the independent-test set 1 and always in the independent-test set 2

\section{Discussion}

In this study, we construct an adrenal gland auto-segmentation framework based on the deep learning, so that the autosegmentation of adrenal glands and periadrenal fat can be rapidly finished. We found that several radiomics features and seven clinical indicators were relatively related to disease prognosis in patients with COVID-19. Next, we established and validated a RN for predicting disease prognosis based on the radiomics features extracted from the onset of the adrenal gland and periadrenal fat CT images combined with clinical indicators. Our study indicated that the auto-segmentation framework could robustly localize the adrenal glands and accurately refine their boundaries, and the radiomics features reflect the microscopic changes in adrenal 
glands and periadrenal fat of patients with COVID-19. Therefore, the RN constructed in combination radiomics features with clinical indicators performed well and could be used to predict the disease prognosis of COVID-19 patients.

Our results suggested that the change of adrenal gland and periadrenal fat on the onset of CT images in severe patients is different from those in moderate patients. Results from autopsies in 10 patients from COVID-19 performed by Zinserling et al. showed that even macroscopic changes of adrenal glands were unremarkable [16]. However, they found inflammation-small proliferations of cells with enlarged light nuclei and mononuclear infiltration, CD3+ and CD8+ in different layers of adrenal glands and their surrounding tissue like periadrenal fat. These changes may be related to direct damage of adrenal glands and indirect changes stimulated by systemic inflammation caused by SARS-CoV-2 or immune state. Firstly, the changes were caused by direct damage done by SARS-CoV-2. The SARS virus has been identified in adrenal cells, suggesting a direct local replication-mediated cytopathic effect of the virus in adrenal tissue [17]. Moreover, virus may cause hemorrhage, necrosis, or thrombosis at the adrenal level [18]. Adrenal lesions due to influenza and other viruses have been described previously [19]. Recent findings have indicated that for the patients with COVID-19, the possibility of venous thrombo-embolism may cause an acute adrenal insufficiency that may be an indicator of making the disease worse [20]. Additionally, the adrenal gland is one of the most highly vascularized organs of the human body, almost every adrenal cell is close to endothelial cells. Therefore, endotheliitis in COVID-19 may further increase the vulnerability of adrenal tissue. However, this needs further study and validation. We hypothesized that the same effect could occur in patients with COVID-19 due to similar virus types. The predicting results in this paper showed that AM's AUC value was slightly better than PM's, but there was no statistical significance. That may indicate that the inner changes in adrenal glands are not different from the changes from periadrenal fat. Further research is needed to confirm the changes in periadrenal fat caused by adrenal gland inflammatory cells' infiltration or direct damage of SARS-CoV-2 [21].

Previous research also reported that rather than direct damaged by SARS-CoV- 2 itself, the change of adrenal gland was believed to be caused by immune overreaction or cytokine storm-inducing endocrinological pathway impaired through a tight binding mechanism of SARS-CoV-2 and ACE2 [22]. Endocrinological pathway impairment may be related to the following aspects. First, ACE2 is highly expressed in adrenals, which serves as the entry receptor for SARS-CoV-2 that is bound to be significantly affected. Although there are no studies have proved it, some works have proposed that the imbalanced ACE/ACE2 axis may mediate tissue repair and heal pathways in the lungs, such as fibrosis after SARS-CoV-2 [23]. Second, the main substrate for ACE2 is angiotensin II. ACE2 acts as a negative regulator of the renin-angiotensin-aldosterone system (RAAS) by converting the active angiotensin and angiotensin II to the inactive angiotensin 1-7 [24]. The adrenal gland is one of the most critical end organs of RAAS that is fundamental in regulating blood pressure, maintaining homeostasis, and is mediated in the organs' inflammatory response. Finally, the SARS virus contains several permutations of amino acid sequences with homology to the antigenic relevant residues of ACTH that will have potential and significant pathophysiological effects due to molecular mimicry [25]. ACTH is a key hormone that regulates the release of autoantibodies from adrenal corticosteroids and could abrogate the adrenal stress response when autoantibodies are binding ACTH. Potential cross-reactivity of antibodies may cause local infiltration with immunocompetent cells in adrenal glands [16]. In summary, these aspects suggest that adrenal cells and surrounding tissue damage in patients with COVID-19 may be caused by a viral infection and further secondary inflammatory plus autoimmune processes located in the adrenal glands.

\section{Conclusions}

we proposed and constructed an adrenal gland auto-segmentation framework based on chest CT images using Al technology. We automatically obtained the ROI from the $\mathrm{CT}$ images through the auto-segmentation framework and inflation algorithm, and then developed and validated a RN which combined the features extracted from the onset CT images with clinical indicators for predicting the disease prognosis of COVID-19. Experiments results showed that CT imaging features of adrenal glands and periadrenal fat have great potential value in predicting the prognosis of COVID-19, and the RN combining these features and clinical factors can be used for preliminary assessment of disease prognosis. To our knowledge, this is the first study using adrenal gland CT parameters as indicators to evaluate the prognosis in patients with COVID-19, especially in the radiomics field. However, there are some limitations in this study. First, we used the chest CT images as data resources. Considering the clinical practicality and radiation to patients, repeating CT scan using professional adrenal glands CT parameters to better observe adrenal lesions is unnecessary. In fact, the main role of CT in examining patients with COVID-19 is to detect and observe pulmonary lesions. Second, different from other studies, we selected the entire organ as ROIs rather than the lesion itself. The periadrenal fat

Page 9/16 
area may imprecise and contain some other indistinguishable tissue ignored by humans. Although there are some deficiencies, our findings validated the potential of radiomics features extracted from adrenal glands and periadrenal fat CT images to be the indicators of COVID-19 prognosis. However, these need to be validated in large-scale prospective studies.

\section{Abbreviations}

LASSO: least absolute shrinkage and selection operator; CM: clinical model AM: adrenal gland model; PM: periadrenal fat model; FM: fusion of adrenal gland and periadrenal fat model; RN: radiomics nomogram model; ROI: region of interest; MAE: mean absolute error; PCR: polymerase chain reaction Al: artificial intelligence; AUC: under the curve; ACTH: adrenocorticotropic hormone; SNS: sympathetic nervous system; ACE2: angiotensin-converting enzyme-2; HSH: Huoshenshan Hospital; MCH: Maternal and Child Health Hospital Optical Valley Branch Hospital; TTH: Taikang Tongji (Wuhan) Hospital; GLCM: gray-level co-occurrence matrix; GLRLM: gray-level run-length matrix; GLSZM: gray-level size zone matrix; GLDM: gray-level dependence matrix; NGTDM: neighboring Gray Tone Difference Matrix; LR: logistic regression; DCA: Decision curve analysis; IL-6: Interleukin 6; L: Iymphocyte count; CRP: C-reactive protein; PCT: procalcitonin; DD:D dimer; AST: glutamic oxaloacetic transaminase; BNP: B-type natriuretic peptide; LDH: lactate dehydrogenase; CK: creatine kinase ; CK-MB: creatine kinase-MB; WBC: white blood cell count; N:neutrophil count; HB: hemoglobin; RBC: red blood cell count; PLT: blood platelet count; PT: prothrombin time; TT :thrombin time; FIB: fibrinogen; BS: blood sugar; ALT: Alanine transaminase; TP: total protein; ALB: albumin; TBil: total bilirubin; D-Bil: direct bilirubin; BUN: blood urea nitrogen; Cre: creatinine; Mb: myoglobin; Tn: troponin; CK: creatine kinase; SEN: sensitivity; SPE: specificity; VIF: variance inflation factor; RAAS: renin-angiotensin-aldosterone system.

\section{Declarations}

\section{Ethics approval and consent to participate}

This multicenter study was approved by the ethics committees of all the participating hospitals (2020, N0.01). Because of its retrospective nature, informed consent forms were not required but all the image and clinical data were anonymized, and the results of the study did not alter any treatment plans for the subjects. The study was performed according to the principles of the declaration of Helsinki.

\section{Consent for publication}

Not applicable

\section{Availability of data and materials}

The datasets used and/or analysed during the current study are available from the corresponding author on reasonable request.

\section{Competing interests}

The authors declare that they have no competing interests

\section{Funding}

The study was supported by the Non-profit Central Research Institute Fund of Chinese Academy of Medical Sciences Q2019PT320003邓; The Science and Technology Foundation of Guizhou Province (QKHPTRC[2019]5803); The Guiyang Science and Technology Project (ZKXM[2020]4); Beijing Medical and Health Foundation (YWJKJJHKYJJ-B20261CS) and Chongqing Science and Health Joint Medical Research Project(2021MSXM052)

\section{Authors' contributions}

First author, Mudan Zhang: design of the work, conducting experiments, analyzing data and writing the manuscript; Co-first author, Xuntao Yin: acquiring data, design of the work, supervising the work and review the manuscript. Yan Zha: design of the work; Xianchun Zeng: Resources; Wuchao Li: statistics analysis; Xiaoyong Zhang: Technology analysis; Jingjing Cui: statistics analysis; 
Zhong Xue: Review \& Editing; Corresponding Author, Chen Liu: Writing - Review \& Editing, Project administration; Verified underlying data. Co-corresponding Author, Rongpin Wang: Writing - Review \& Editing, Project administration; Verified underlying data.

\section{Acknowledgements}

The study was supported by the Non-profit Central Research Institute Fund of Chinese Academy of Medical Sciences \2019PT320003区; The Science and Technology Foundation of Guizhou Province (QKHPTRC[2019]5803); The Guiyang Science and Technology Project (ZKXM[2020]4); Beijing Medical and Health Foundation (YWJKJJHKYJJ-B20261CS) and Chongqing Science and Health Joint Medical Research Project(2021MSXM052)

\section{Prior Presentation}

This manuscript has been preprint in medRxiv (doi: https://doi.org/10.1101/2021.01.03.21249183) but not been reviewed, published or presented elsewhere in part or in entirety and is not under consideration by another journal.

\section{Declaration of interests}

The authors have declared that no conflict of interest exists.

\section{References}

1. Tian M, Long Y, Hong Y, et al. The treatment and follow-up of'recurrence' with discharged COVID - 19 patients: data from Guizhou, China. Environmental Microbiology. 2020;22(8):3588-92.

2. Giorgia Guglielmi. Fast coronavirus tests: what they can and can't do. Nature. 2020; 585, 496-498.

3. Zhang K. Clinically Applicable AI System for Accurate Diagnosis, Quantitative Measurements and Prognosis of COVID-19 Pneumonia Using Computed Tomography. CELL. 2020; 181(6): 1423-1433.

4. Ting DSW, Carin L, Dzau V, et al. Digital technology and COVID-19. Nat Med. 2020. 26: 459-461

5. Agarwal S, Agarwal SK. Endocrine changes in SARS-CoV-2 patients and lessons from SARS-CoV. Postgrad Med J. 2020;96(1137):412-6.

6. Bellastella G, Maiorino MI, and Esposito K. Endocrine complications of COVID-19: what happens to the thyroid and adrenal glands? Journal of Endocrinological Investigation. 2020;43(8):1169-70.

7. Waldemar Kanczkowski, and Mariko Sue SRB, 2. The adrenal gland microenvironment in health, disease and during regeneration. 2017. 16(3):251-265.

8. Pal R, and Banerjee M. COVID-19 and the endocrine system: exploring the unexplored. Journal of Endocrinological Investigation. 2020;43(7):1027-31.

9. Peter Sever, Sebastian L Johnston. The Renin-Angiotensin system and SARS-CoV-2 infection: A role for the ACE2 receptor? [published online May 13, 2020]. J Renin Angiotensin Aldosterone Syst. doi: 10.1177/1470320320926911.

10. Berger I, Werdermann M, Bornstein SR,et al.. The adrenal gland in stress - Adaptation on a cellular level. The Journal of Steroid Biochemistry and Molecular Biology. 2019;190:198-206.

11. Gillies RJ, Kinahan PE, Hricak H. Radiomics: Images Are More than Pictures, They Are Data. Radiology. 2016. 278(2):563-77.

12. J. Wang, Yiming Bao, Yaofeng Wen et al."Prior-Attention Residual Learning for More Discriminative COVID-19 Screening in CT Images," in IEEE Transactions on Medical Imaging, vol. 39, no. 8, pp. 2572-2583, Aug.2020, doi: 10.1109/TMI.2020.2994908.

13. Han, M., Yao, G., Zhang, W et al. Segmentation of CT Thoracic Organs by Multi-resolution VB-nets. SegTHOR@ISBI. 2019. Corpus ID: 139100153

14. Hua R, Huo Q, Gao Y, et al. Segmenting Brain Tumor Using Cascaded V-Nets in Multimodal MR Images. [published online Feb 14, 2020] Frontiers in Computational Neuroscience. doi: 10.3389/fncom.2020.00009.

15. van Griethuysen, J. J. M., Fedorov, A., Parmar, C.,et al. Computational Radiomics System to Decode the Radiographic Phenotype. Cancer Research, 2017, 77(21), e104-e107. 
16. Zinserling VA, Semenova NY, Markov AG, et al. Inflammatory Cell Infiltration of Adrenals in COVID-19. Horm Metab Res. 2020. 52(9):639-641.

17. Gu J, and Korteweg C. Pathology and Pathogenesis of Severe Acute Respiratory Syndrome. The American Journal of Pathology. 2007;170(4):1136-47.

18. Henry BM, Vikse J, Benoit S, et al. Hyperinflammation and derangement of renin-angiotensin-aldosterone system in COVID-19: A novel hypothesis for clinically suspected hypercoagulopathy and microvascular immunothrombosis. Clin Chim Acta. 2020;507:167-73.

19. Zinserling AV, Aksenov OA, Melnikova VF et al. Extrapulmonary lesions in influenza. Tohoku J Exp Med. 1983. 140(3):259-72.

20. Tseng YH, Yang RC, Lu TS. Two hits to the renin-angiotensin system may play a key role in severe COVID-19. Kaohsiung J Med Sci. 2020;36(6):389-92.

21. Battisti S, Pedone C, Napoli N et al. Computed Tomography Highlights Increased Visceral Adiposity Associated With Critical Illness in COVID-19. Diabetes Care. 2020;43(10):e129-e30.

22. Datta PK, Liu F, Fischer T, et al. SARS-CoV-2 pandemic and research gaps: Understanding SARS-CoV-2 interaction with the ACE2 receptor and implications for therapy. Theranostics. 2020;10(16):7448-64.

23. Delpino MV, Quarleri J. SARS-CoV-2 Pathogenesis: Imbalance in the Renin-Angiotensin System Favors Lung Fibrosis. Front Cell Infect Microbiol. 2020;10:340.

24. Cheng $\mathrm{H}$, Wang $\mathrm{Y}$, and Wang GQ. Organ - protective effect of angiotensin - converting enzyme 2 and its effect on the prognosis of COVID - 19. Journal of Medical Virology. 2020;92(7):726-30.

25. Pal R. COVID-19, hypothalamo-pituitary-adrenal axis and clinical implications. Endocrine. 2020;68(2):251-2.

\section{Figures}




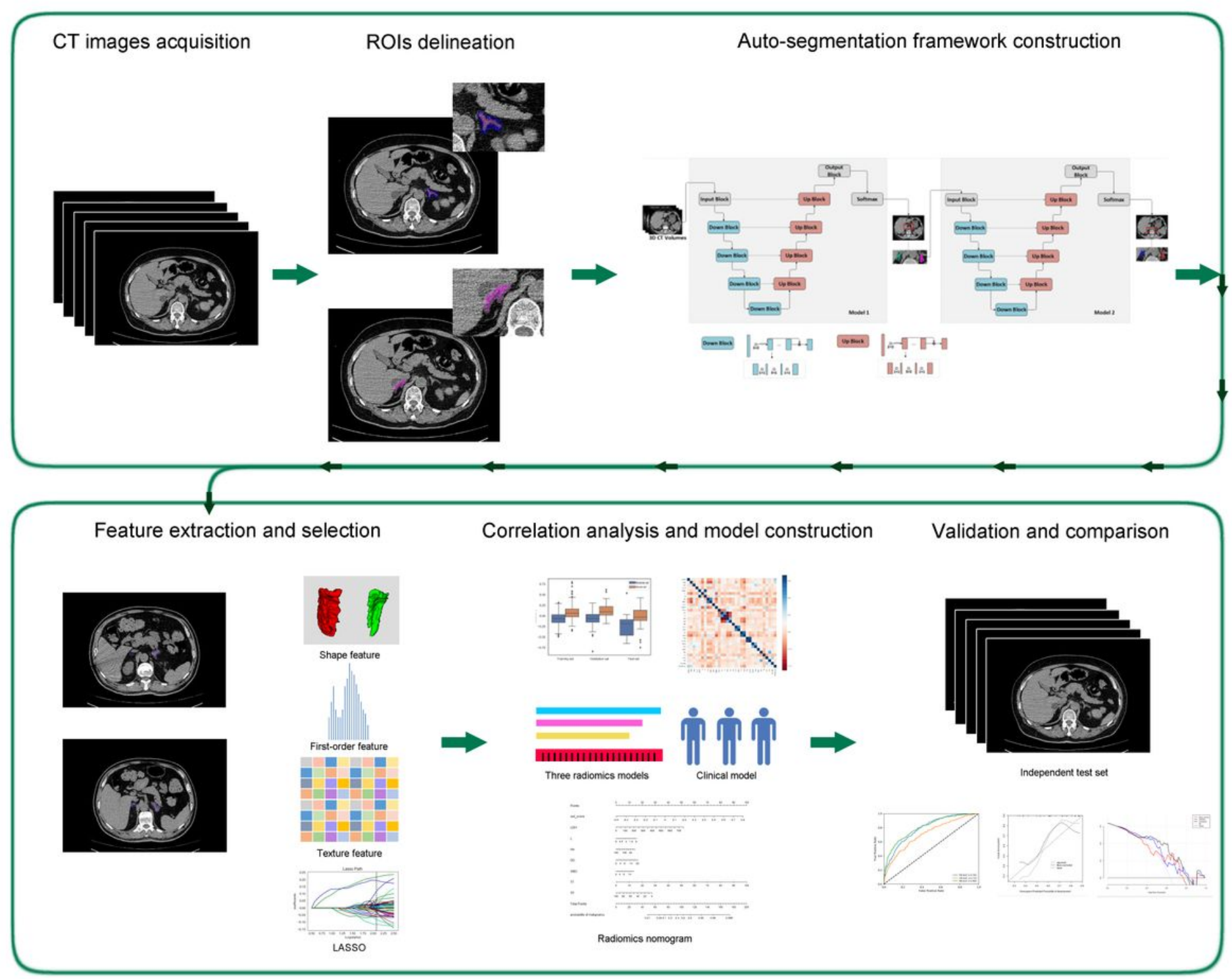

Figure 1

The workflow of study. 


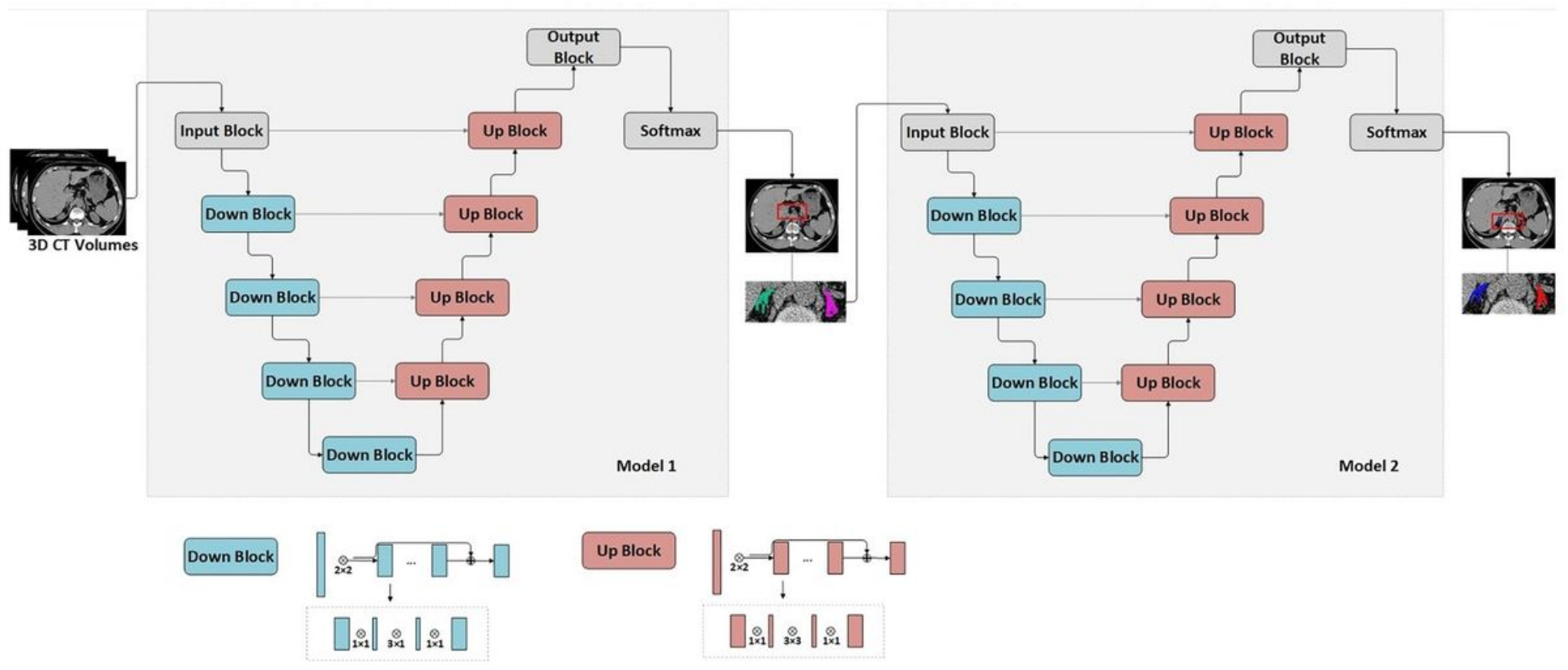

Figure 2

The framework of the used V-net. 
A

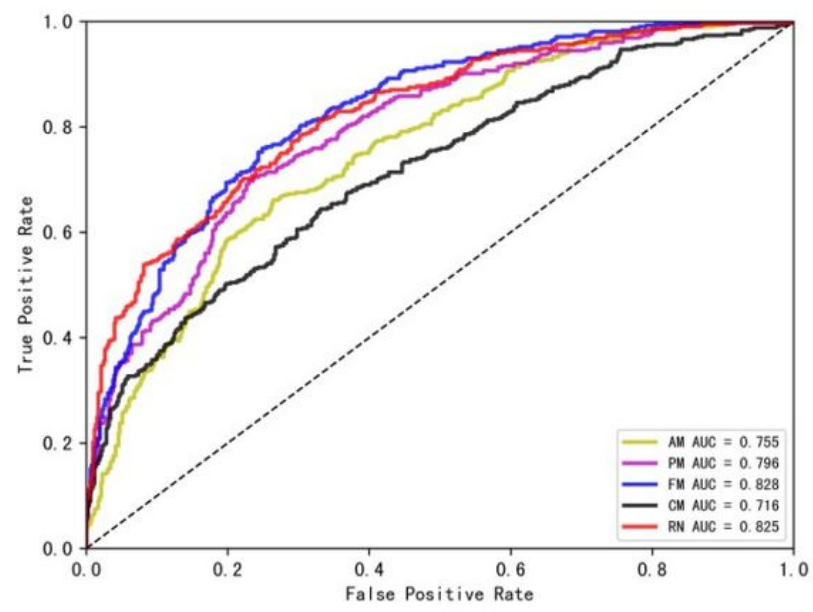

C

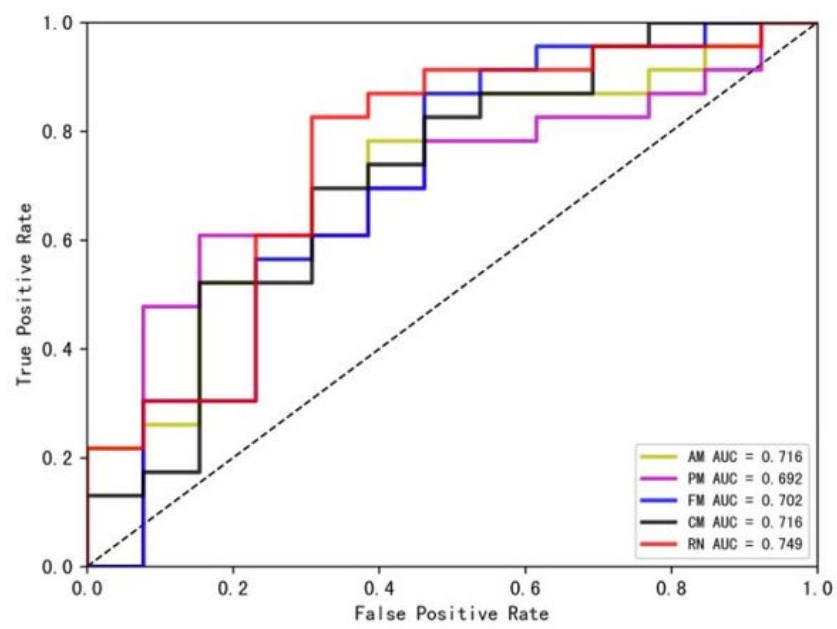

B

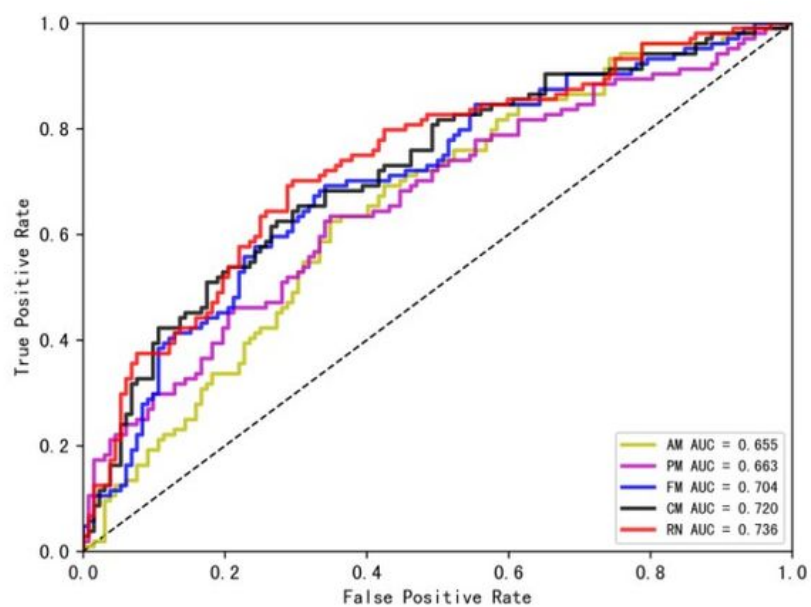

D



Figure 3

ROC curves in training (A), validation (B), independent test set 1 (C) and independent test set 2 (D). AM = adrenal gland model; PM = periadrenal fat model; $\mathrm{FM}=$ fusion of adrenal gland and periadrenal fat model; $\mathrm{CM}=$ clinical model; $\mathrm{RN}=$ radiomics nomogram 
Points

RadScore

Age

$\mathrm{LDH}$

L

$\mathrm{Hb}$

DD

WBC

TT

TP

Total Points

probability of malignancy
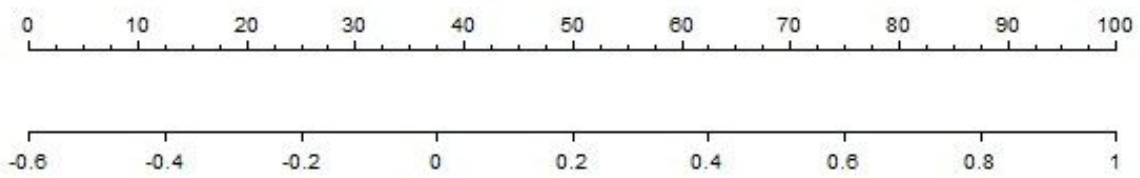

тाтाтाтा

$100 \quad 40$



तागाणामापा

$\begin{array}{llll}6 & 4 & 2 & 0\end{array}$

$18080 \quad 0$



ता पा 1 1 1 1 1

$\begin{array}{lllll}0 & 4 & 8 & 12 & 18\end{array}$

\begin{tabular}{llllllllll}
\hline 0 & 10 & 20 & 30 & 40 & 50 & 60 & 70 & 80 & 90
\end{tabular}

तापापापात

$100 \quad 60 \quad 20$

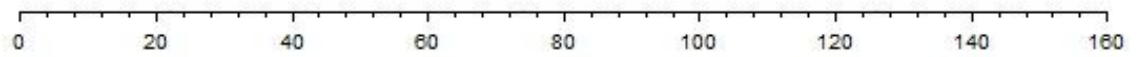

\section{Figure 4}

Radiomics nomogram developed in training set with radiomics features, lactate dehydrogenase (LDH), lymphocyte count $(L)$, hemoglobin (Hb), D dimer (DD), white blood cells count (WBC), thrombin time (TT) and total protein (TP). Points are assigned for each variable by drawing a line upward from the corresponding variable to the Points line. The sum of points plotted on the total Points line corresponds with the severity of patients with COVID-19.

\section{Supplementary Files}

This is a list of supplementary files associated with this preprint. Click to download.

- 20211008Addionalfile.pdf

- Supplementaryfigure4BOX.jpg

- Supplementaryfigure5abias.tif

- Supplementaryfigure6bdecision.tif

- supplementaryfigure2segmentation.tif

- supplementaryfigure3heatmap.tif 